typeset using JPSJ.sty $<$ ver.1.0b $>$

\title{
Spin Excitations in the Two-leg Ladder Antiferromagnet $\mathrm{CaV}_{2} \mathrm{O}_{5}$
}

\author{
Tetsuo Ohama ${ }^{1,2, *}$, Masahiko IsoBe $^{1}$ and Yutaka UedA ${ }^{1}$ \\ ${ }^{1}$ Institute for Solid State Physics, University of Tokyo, Kashiwanoha, Kashiwa, 277-6581 \\ ${ }^{2}$ Department of Physics, Faculty of Science, Chiba University, Inage-ku, Chiba, 263-8522
}

(Received )

\begin{abstract}
The nuclear spin-lattice relaxation rate at the $\mathrm{V}$ sites in $\mathrm{CaV}_{2} \mathrm{O}_{5}$ was measured. The relaxation rate $\left(1 / T_{1}\right)$ in the two-leg ladder was calculated with a strong-coupling expansion $\left(J_{\perp} \gg J_{\|}\right)$ and the result was compared with the experimental data. The comparison of the temperature dependence shows a good agreement and provides an estimate of both $J_{\perp}$ and $J_{\|}$as well as the energy gap $\Delta$. The magnetic field dependence of $1 / T_{1}$ is also reproduced with this approximation in the temperature range $T \lesssim \Delta / 4$. It is found that the magnon-magnon collision, which leads to a diffusive behavior, is negligible in this temperature range. The effect of inter-ladder coupling is investigated and found to be weak in $\mathrm{CaV}_{2} \mathrm{O}_{5}$. Thus the strong-coupling expansion calculation agrees satisfactorily with the experimental data, but a discrepancy between the exchange parameters deduced from the magnetic susceptibility and $1 / T_{1}$ is found.
\end{abstract}

KEYWORDS: $\mathrm{CaV}_{2} \mathrm{O}_{5}, \mathrm{NMR}$, spin ladder, spin diffusion, nuclear spin relaxation

\section{$\S 1 . \quad$ Introduction}

The discovery of high- $T_{\mathrm{C}}$ superconductivity in doped two-dimensional antiferromagnets renewed interest in quantum antiferromagnets. In particular, spin- $\frac{1}{2}$ two-leg ladder antiferromagnets have been extensively studied, being motivated by a theoretical prediction of the quantum disordered ground state with an energy gap and possible superconductivity. ${ }^{1)}$ Actually, the energy gap was experimentally observed in $\mathrm{SrCu}_{2} \mathrm{O}_{3} .{ }^{2}{ }^{2}$ Moreover, superconductivity was discovered in $\mathrm{Sr}_{14-x} \mathrm{Ca}_{x} \mathrm{Cu}_{24} \mathrm{O}_{41}$ under high pressure. ${ }^{3)}$

The low-energy excitations in such gapful spin systems have not been fully understood yet. One problem is a significant discrepancy between the energy gaps determined from the magnetic susceptibility and the nuclear spinlattice relaxation rate $1 / T_{1}$. For the two-leg ladder antiferromagnet $\mathrm{SrCu}_{2} \mathrm{O}_{3}, 1 / T_{1}$ suggests a much larger gap $\Delta \sim 700 \mathrm{~K}$ than $\Delta \sim 420 \mathrm{~K}$ by the susceptibility. ${ }^{2}{ }^{4)}$ Itoh et al. surveyed the data of the susceptibility and $1 / T_{1}$ of many gapful quantum paramagnets and pointed out that this discrepancy is observed widely in one-dimensional gapful systems such as Haldane-gap and two-leg ladder antiferromagnets. ${ }^{5)}$ Kishine and Fukuyama calculated $1 / T_{1}$ with the Majorana fermion representation of the two-leg ladders. ${ }^{6}$ ) They found a dominant contribution with three times larger energy gap than the lowest excitation and conclude that this is responsible for the discrepancy. Sachdev and Damle developed a semiclassical continuum theory for one-dimensional gapful systems and obtained diffusive behavior in the long-time spin correlation. ${ }^{7,8)}$ They showed that $1 / T_{1}$ is not proportional to $\mathrm{e}^{-\Delta / T}$ but $\mathrm{e}^{-3 \Delta / 2 T}$ at sufficient but not too low temperatures. The diffusive behavior was actually observed

* Present Address: Department of Physics, Faculty of Science, Chiba University, Chiba, 263-8522.

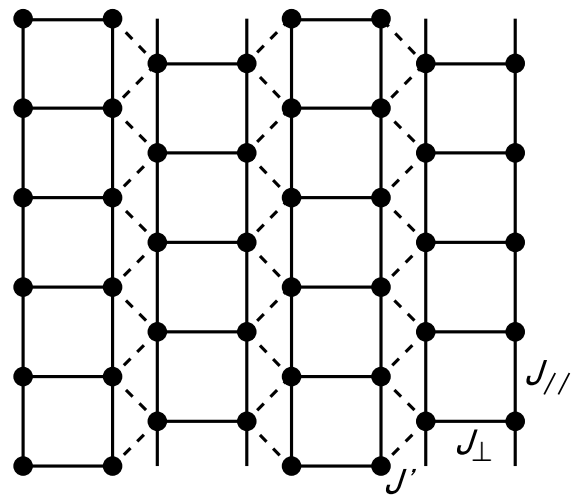

Fig. 1. Schematic drawing of dominant exchange interactions in $\mathrm{CaV}_{2} \mathrm{O}_{5}$ : solid lines for intra-ladder couplings $J_{\|}$and $J_{\perp}$, and dashed lines for inter-ladder coupling $J^{\prime}$.

in magnetic field dependence of $1 / T_{1}$ in the Haldane-gap antiferromagnet $\mathrm{AgVP}_{2} \mathrm{~S}_{6}{ }^{10)}$ and another gapful system $(\mathrm{VO})_{2} \mathrm{P}_{2} \mathrm{O}_{7} \cdot{ }^{11,12)}$ These theoretical arguments suggest that the intrinsic energy gap is unique and that the discrepancy was observed because the data fitting was done at too high temperatures.

The discrepancy between the energy gaps was also observed $^{13)}$ in another two-leg ladder antiferromagnet $\mathrm{CaV}_{2} \mathrm{O}_{5} \cdot{ }^{14,15)} 1 / T_{1}$ at the $\mathrm{V}$ sites indicates activated temperature dependence with an energy gap of $616 \mathrm{~K}$. On the other hand, somewhat smaller gap $\sim 460 \mathrm{~K}$ was suggested from the NMR shift, although the data accuracy was insufficient.

In this compound, one $3 d$ electron per $\mathrm{V}$ site occupies the $d_{x y}$-like orbital in a $\mathrm{VO}_{5}$ pyramid, ${ }^{16)}$ which forms $\mathrm{V}_{2} \mathrm{O}_{5}$ layers. Dominant exchange interactions between electronic spins at the $\mathrm{V}$ sites are schematically shown in Fig. 1: two exchange interactions, $J_{\|}$and $J_{\perp}$, between corner-sharing $\mathrm{VO}_{5}$ pyramids form two-leg ladders and 


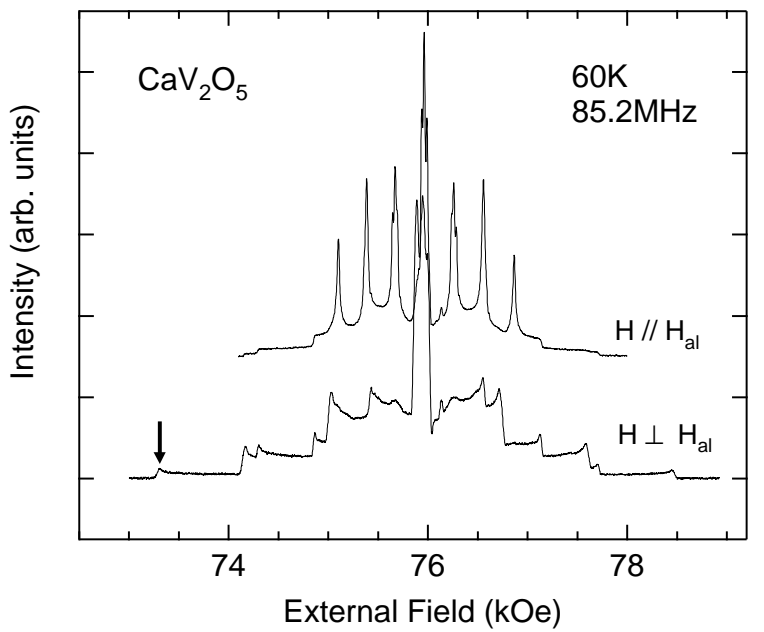

Fig. 2. ${ }^{51} \mathrm{~V}$ NMR spectra at $60 \mathrm{~K}$. Satellite transition used in $1 / T_{1}$ measurements is shown by arrow.

the other between edge-sharing pyramids is frustrating inter-ladder coupling $J^{\prime}$. These exchange interactions form a trellis lattice similarly to $\mathrm{SrCu}_{2} \mathrm{O}_{3}$. Onoda and Nishiguchi reported that the temperature dependence of the magnetic susceptibility can be fitted well by the isolated dimer model with the intra-dimer coupling $664 \mathrm{~K},{ }^{15)}$ suggesting anisotropic exchange interactions near the strong-coupling limit $\left(J_{\perp} \gg J_{\|}\right)$. Miyahara et al. compared the experimental data of the susceptibility to quantum Monte Carlo simulations for the trellis lattice. ${ }^{17)}$ They also concluded that $\mathrm{CaV}_{2} \mathrm{O}_{5}$ is near the isolated dimers: the intra-ladder exchange along the rung is dominant $J_{\perp} \sim 670 \mathrm{~K}$, and the others are in the range of $0<J_{\|}<200 \mathrm{~K}$, and $J_{\|}+J^{\prime} \sim 110 \mathrm{~K}$. An ab initio calculation of the exchange parameters was performed using the LDA $+U$ method. ${ }^{18,19)}$ Their result that $J_{\perp} \sim 608$ $\mathrm{K}, J_{\|} \sim 122 \mathrm{~K}$, and $J^{\prime} \sim-28 \mathrm{~K}$ agrees with the quantum Monte Carlo simulation.

We report in this article $1 / T_{1}$ measurements at the $\mathrm{V}$ sites in $\mathrm{CaV}_{2} \mathrm{O}_{5}$ with a powder sample. The dependence of $1 / T_{1}$ on the temperature and magnetic field was measured in a wide temperature range to compare it to theories in detail. We calculate $1 / T_{1}$ for the twoleg ladder with the strong-coupling expansion in $\S 3$ and compare the result with the experimental data in $\S 4$. We estimate the exchange parameters from the temperature dependence. Next we compare the magnetic field dependence and investigate whether the diffusive behavior is observed in $\mathrm{CaV}_{2} \mathrm{O}_{5}$. Also we examine whether the magnetic susceptibility can be explained consistently with the exchange parameters deduced from the analysis of $1 / T_{1}$.

\section{§2. Experimental}

The powder sample used in this study is the same as the previous NMR shift measurement. ${ }^{16)}$ It was magnetically aligned and was found by an X-ray diffraction measurement that the alignment field $\left(H_{\mathrm{al}}\right)$ was distributed in the $a-b$ plane and mainly along the $b$ axis.
We measured $1 / T_{1}$ by observing the spin-echo recovery of the third satellite transition after an inversion pulse with the magnetic field $(H)$ along the maximum principal axis of the electrical field gradient at the $\mathrm{V}$ sites. At the magnetic field of this satellite transition, shown in ${ }^{51} \mathrm{~V}$ NMR spectra ${ }^{16)}$ in Fig.2, the observed nuclear magnetization recovery can be fit well by the analytical formula of the nuclear relaxation for the uniaxial electrical field gradient $(\eta=0)$ without cross relaxation between the nuclear Zeeman levels ${ }^{20)}$

$$
\begin{aligned}
\frac{M(t)}{M_{0}}= & 1-C\left[\frac{1}{84} \mathrm{e}^{-t / T_{1}}+\frac{3}{28} \mathrm{e}^{-3 t / T_{1}}\right. \\
& +\frac{3}{11} \mathrm{e}^{-6 t / T_{1}}+\frac{25}{77} \mathrm{e}^{-10 t / T_{1}}+\frac{75}{364} \mathrm{e}^{-15 t / T_{1}} \\
& \left.+\frac{3}{44} \mathrm{e}^{-21 t / T_{1}}+\frac{4}{429} \mathrm{e}^{-28 t / T_{1}}\right]
\end{aligned}
$$

We thereby obtain $1 / T_{1}$ with good accuracy. At the magnetic field of the other satellite transition, the spinecho signal contains signals of other satellite transition or for other magnetic field directions, thus the precise measurement of $1 / T_{1}$ is not achievable.

\section{$\S 3 . \quad 1 / T_{1}$ in Strong-coupling Two-leg Ladders}

In this section we calculate $1 / T_{1}$ in two-leg ladders with a strong-coupling expansion to analyze the experimental data. Sagi and Affleck recently developed a theory of nuclear relaxation in the Haldane-gap antiferromagnet, ${ }^{21)}$ which has an excitation spectrum similar to the anisotropic two-leg ladder near the strong-coupling limit. We here calculate $1 / T_{1}$ in the two-leg ladder following their arguments. Difference in the hyperfine interaction between the Haldane-gap and two-leg ladder antiferromanets, that is, the coupling of nuclear spins to the excitations is essential. Further, we also calculate $1 / T_{1}$ for a trellis lattice including the inter-ladder coupling.

In general, $1 / T_{1}$ due to the fluctuating hyperfine field $\boldsymbol{H}^{\mathrm{hf}}(t)$ is given by

$$
\frac{1}{T_{1}}=\frac{\gamma^{2}}{2} \int_{-\infty}^{\infty} d t\left\langle\left\{H_{+}^{\mathrm{hf}}(t) H_{-}^{\mathrm{hf}}(0)\right\}\right\rangle_{\beta} \mathrm{e}^{i \omega_{\mathrm{N}} t}
$$

where $\gamma$ is the nuclear gyromagnetic ratio, $\omega_{\mathrm{N}} \equiv \gamma H$, $\{A B\} \equiv(A B+B A) / 2$, and $\langle\ldots\rangle_{\beta}$ is the thermal average. ${ }^{22)}$ Here we take the $z$ axis along the magnetic field. For the magnetic nuclear sites, we can neglect the offdiagonal part of the hyperfine interaction as

$$
\mathcal{H}_{\mathrm{hf}}=\frac{A_{\perp}}{2}\left(I_{+} S_{-}+I_{-} S_{+}\right)+A_{\|} I_{z} S_{z}
$$

then $1 / T_{1}$ is

$$
\frac{1}{T_{1}}=\frac{A_{\perp}^{2}}{2 \hbar^{2}} \int_{-\infty}^{\infty} d t\left\langle\left\{S_{+}(t) S_{-}(0)\right\}\right\rangle_{\beta} \mathrm{e}^{i \omega_{\mathrm{N}} t} .
$$

For the two-leg ladder antiferromagnet, the low-lying excitations consist of triplet "magnons". ${ }^{23,24)}$ The main nuclear relaxation process is the scattering of a thermally excited magnon by a nuclear spin (the Raman process). These magnons are not real bosons, since one cannot excite two magnons on the same rung at the same time, 
but at low temperatures $T \ll \Delta$ where the number of excited magnons is small, we can consider the magnons as independent bosons. Then $1 / T_{1}$ is

$$
\begin{aligned}
\frac{1}{T_{1}}= & \frac{A_{\perp}^{2}}{2 \hbar^{2}} \sum_{k, k^{\prime}} \sum_{\sigma, \sigma^{\prime}}\left|\left\langle k^{\prime}, \sigma^{\prime}\left|S_{+}\right| k, \sigma\right\rangle\right|^{2}\left(\frac{\mathrm{e}^{-\beta \epsilon_{k \sigma}}+\mathrm{e}^{-\beta \epsilon_{k^{\prime} \sigma^{\prime}}}}{2}\right) \\
& \times \int_{-\infty}^{\infty} d t \mathrm{e}^{i t\left(\epsilon_{k \sigma}-\epsilon_{k^{\prime} \sigma^{\prime}}-\hbar \omega_{\mathrm{N}}\right) / \hbar},
\end{aligned}
$$

where $|k \sigma\rangle$ is a one-magnon state with momentum $k$ and $z$ component of spin $\sigma=-1,0,1$, and $\beta \equiv 1 / T$.

We first consider the two-leg ladder

$$
\mathcal{H}=\sum_{r=1}^{N} J_{\perp} \boldsymbol{S}_{r}^{\mathrm{L}} \cdot \boldsymbol{S}_{r}^{\mathrm{R}}+\sum_{r=1}^{N} J_{\|}\left(\boldsymbol{S}_{r}^{\mathrm{L}} \cdot \boldsymbol{S}_{r+1}^{\mathrm{L}}+\boldsymbol{S}_{r}^{\mathrm{R}} \cdot \boldsymbol{S}_{r+1}^{\mathrm{R}}\right) .
$$

in the strong-coupling expansion; ${ }^{23,25)} \boldsymbol{S}_{r}^{\mathrm{L}}$ and $\boldsymbol{S}_{r}^{\mathrm{R}}$ are spin operators at the left $(\mathrm{L})$ and right $(\mathrm{R})$-hand sites respectively on each rung $r$ of the ladder. When $J_{\|}=0$, the system consists of $N$ noninteracting dimers and the eigenstates are direct products of singlet or triplet states of the dimers. To the first order in $J_{\|} / J_{\perp}$, the lowest excited states are given by Bloch states

$$
|k \sigma\rangle=\frac{1}{\sqrt{N}} \sum_{r=1}^{N} \mathrm{e}^{i k r}\left|s \ldots t_{r}^{\sigma} \ldots s\right\rangle,
$$

where the $r$ th rung is excited to a triplet with the $z$ component of spin $\sigma$, and have a cosine dispersion

$$
\epsilon_{k}=J_{\perp}+J_{\|} \cos k .
$$

Then we obtain the matrix element

$$
\begin{aligned}
\left|\left\langle k^{\prime}, \sigma+1\left|S_{r+}^{L}\right| k \sigma\right\rangle\right|^{2} & =\frac{1}{N^{2}}\left|\mathrm{e}^{i\left(k-k^{\prime}\right) r}\left\langle t_{r}^{\sigma+1}\left|S_{r+}^{L}\right| t_{r}^{\sigma}\right\rangle\right|^{2} \\
& =\frac{1}{2 N^{2}},
\end{aligned}
$$

and thus

$$
\begin{aligned}
\frac{1}{T_{1}}= & \frac{A_{\perp}^{2}}{4 \hbar^{2}} \frac{1}{N^{2}} \sum_{k, k^{\prime}}\left(\mathrm{e}^{-\beta \epsilon_{k}}+\mathrm{e}^{-\beta\left(\epsilon_{k}+h\right)}\right) \\
& \times 2 \pi \hbar \delta\left(\frac{\epsilon_{k}-\epsilon_{k^{\prime}}+h}{\hbar}-\omega_{\mathrm{N}}\right),
\end{aligned}
$$

where $h \equiv g \mu_{B} H$. Since $h \gg \hbar \omega_{\mathrm{N}}$, the Zeeman splitting of the magnon bands is essential and $\omega_{N}$ is negligible. Replacing the sum by integral

$$
\frac{1}{N} \sum_{k} \ldots \rightarrow \int_{0}^{\infty} d \epsilon \rho(\epsilon) \ldots
$$

we obtain

$$
\frac{1}{T_{1}}=\frac{\pi A_{\perp}^{2}}{2 \hbar} \int_{0}^{\infty} d \epsilon \rho(\epsilon) \rho(\epsilon+h) \mathrm{e}^{-\beta \epsilon}\left(1+\mathrm{e}^{-\beta h}\right),
$$

where $\rho(\epsilon)$ is the magnon density. For the cosine dispersion eq. (3.7), we obtain

$$
\begin{aligned}
\frac{1}{T_{1}}= & \frac{A_{\perp}^{2}}{2 \pi \hbar J_{\|}} \mathrm{e}^{-\beta \Delta}\left(1+\mathrm{e}^{-\beta h}\right) \\
& \times \int_{-1}^{1-h / J_{\|}} d x \frac{\mathrm{e}^{-\beta J_{\|}(1+x)}}{\sqrt{1-x^{2}} \sqrt{1-\left(x+h / J_{\|}\right)^{2}}},
\end{aligned}
$$

where $\Delta=J_{\perp}-J_{\|}$. If we expand the dispersion around the band bottom $k=\pi$ as

$$
\epsilon_{k}=\Delta+\frac{c^{2}}{2 \Delta}(\pi-|k|)^{2}
$$

where the magnon velocity $c=\sqrt{\Delta J_{\|}}$, we obtain

$$
\frac{1}{T_{1}} \approx \frac{A_{\perp}^{2}}{2 \pi \hbar J_{\|}} \mathrm{e}^{-\beta \Delta} \cosh \frac{\beta h}{2} K_{0}\left(\frac{\beta h}{2}\right),
$$

where $K_{0}(x)$ is the modified Bessel function of the second kind. Troyer et al. obtained a similar result ${ }^{24)}$ but its magnetic-field dependence is unrealistic because they neglected the Zeeman splitting of the magnon bands.

Next we consider the trellis lattice including the interladder coupling $J^{\prime}$. We have to treat a two dimensional magnon dispersion. To the first order in $J_{\|} / J_{\perp}$ and $J^{\prime} / J_{\perp}$, the triplet magnon band splits into two branches as $^{17)}$

$$
\epsilon\left(k_{\perp}, k_{\|}\right)=J_{\perp}+J_{\|} \cos k_{\|} \pm\left|J^{\prime}\right| \cos \frac{k_{\perp}}{2} \cos \frac{k_{\|}}{2},
$$

and these states are given by Bloch states similarly to eq. (3.6). Then we obtain the matrix elements

$$
\left|\left\langle\boldsymbol{k}^{\prime}, \alpha^{\prime}, \sigma+1\left|S_{r+}^{L}\right| \boldsymbol{k}, \alpha, \sigma\right\rangle\right|^{2}=\frac{1}{2 N^{4}},
$$

where $\boldsymbol{k}=\left(k_{\perp}, k_{\|}\right)$and $\alpha$ indicates one of the two branches. $1 / T_{1}$ is given by

$$
\begin{aligned}
\frac{1}{T_{1}}= & \frac{A_{\perp}^{2}}{4 \hbar^{2}} \frac{1}{N^{4}} \sum_{\boldsymbol{k}, k^{\prime}} \sum_{\alpha, \alpha^{\prime}}\left(\mathrm{e}^{-\beta \epsilon(\boldsymbol{k}, \alpha)}+\mathrm{e}^{\left.-\beta \epsilon\left(\boldsymbol{k}^{\prime}, \alpha^{\prime}\right)+h\right)}\right) \\
& \times 2 \pi \hbar \delta\left(\frac{\epsilon(\boldsymbol{k}, \alpha)-\epsilon\left(\boldsymbol{k}^{\prime}, \alpha^{\prime}\right)+h}{\hbar}-\omega_{\mathrm{N}}\right),
\end{aligned}
$$

and using the magnon density we finally obtain the same expression as eq. (3.10).

For both the two-leg ladder and the trellis lattice, $1 / T_{1}$ depends on not only the energy gap $\Delta$ but also the whole magnon density, thereby we can estimate the exchange parameters from the analysis of $1 / T_{1}$.

\section{§4. Experimental Results and Discussion}

In this section we present the experimental results and compare it with the theoretical calculation.

\subsection{Temperature Dependence of $1 / T_{1}$}

Figure 3 shows the temperature dependence of $1 / T_{1}$ measured with the magnetic field of $73 \mathrm{kOe}$. We observed activated temperature dependence down to $40 \mathrm{~K}$. This clearly indicates the singlet ground state, and thus contradicts a $\mu \mathrm{SR}$ measurement which suggests spin freezing below $\sim 50 \mathrm{~K}^{26}$ ) This is most likely an impurity effect caused by incident muons.

We first compare the observed temperature dependence of $1 / T_{1}$ with a numerical calculation of eq.(3.11) for the two-leg ladder without the inter-ladder coupling. Using $A_{\perp}=\sqrt{\left(A_{x}^{2}+A_{y}^{2}\right) / 2} \sim 5.9 \times 10^{-3} \mathrm{~K}$, ${ }^{16)}$ we can fit the $1 / T_{1}$ data well with $J_{\perp} \sim 655(5) \mathrm{K}, J_{\|} \sim 93(5)$ $\mathrm{K}$, and consequently $\Delta \sim 562(5) \mathrm{K}$. This confirms the anisotropic exchange interactions $J_{\perp} \gg J_{\|}$. The calculated temperature dependence is in good agreement 


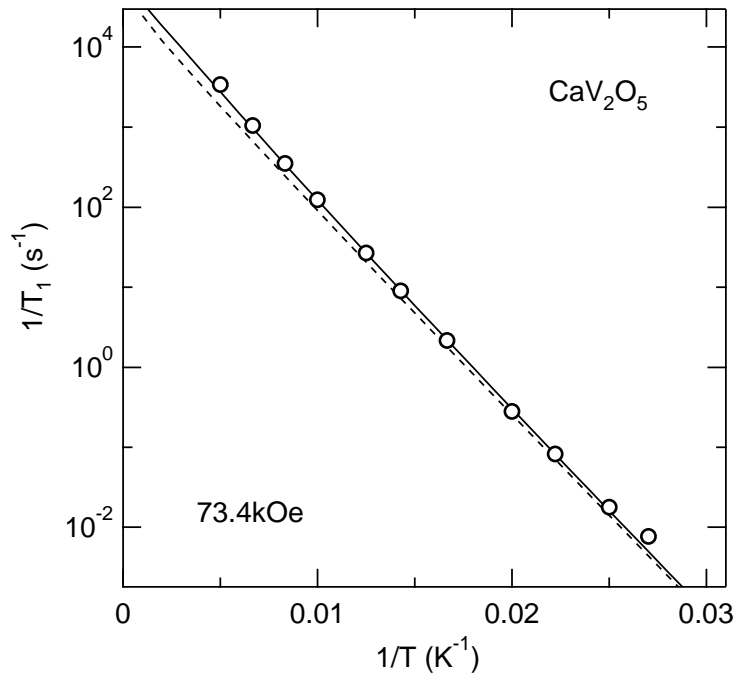

Fig. 3. Temperature dependence of $1 / T_{1}$. Solid line shows a fit to the strong-coupling expansion eq. (3.11) (the cosine dispersion). Dashed line is calculated with the parabolic dispersion with the same $J_{\perp}$ and $J_{\|}$.

with the data except $35 \mathrm{~K}$ and above $150 \mathrm{~K}$ as shown in Fig. 3. At the low temperatures the deviation is most likely due to magnetic impurities and defects. At high temperatures we expect that the independent magnon approximation is not applicable.

\subsection{Magnetic field dependence of $1 / T_{1}$}

Figure 4 shows the magnetic field dependence of $1 / T_{1}$; we found weak but clear dependence. We calculate the magnetic field dependence numerically with eq. (3.11) using the exchange parameters determined in the previous section $J_{\perp}=655 \mathrm{~K}, J_{\|}=93 \mathrm{~K}$. This calculation agrees with the experimental data as shown in Fig. 4.

Recently Sachdev and Dample developed a semiclassical continuum theory for one-dimensional gapful antiferromagnets. ${ }^{7,9)}$ They considered magnon-magnon collision and found a diffusive behavior in $1 / T_{1}$. They found that $1 / T_{1} \propto \mathrm{e}^{-3 \beta \Delta / 2}$ not $\propto \mathrm{e}^{-\beta \Delta}$ at 'not too low' temperatures. They claimed that this 'large gap' $3 \Delta / 2$ explains the discrepancy between energy gaps determined from $1 / T_{1}$ and magnetic susceptibility in onedimensional gapful antiferromagnets. At low temperatures this theory agrees with the independent magnon approximation eq. (3.13) but at high temperatures or at low magnetic fields the effect of magnon-magnon collision appears. We compare these theories and investigate whether the magnon-magnon collision is important in the temperature and magnetic field ranges of our measurement in $\mathrm{CaV}_{2} \mathrm{O}_{5}$.

They obtain the dynamical structure factor for a parabolic dispersion $\epsilon_{k}=\Delta+\left(c^{2} / 2 \Delta\right)(\pi-|k|)^{2}$,

$$
\begin{aligned}
S_{\mp, \pm}\left(\omega_{\mathrm{N}}\right)= & \frac{2\left(\rho_{0}+\rho_{\mp 1}\right)}{c} \sqrt{\frac{2 \Delta}{\pi T}}\left\{\ln \left(L_{t} T\right)\right. \\
& \left.+\Phi_{2}\left(\sqrt{\pi}\left|\hbar \omega_{\mathrm{N}} \pm h\right| L_{t}\right)\right\},
\end{aligned}
$$

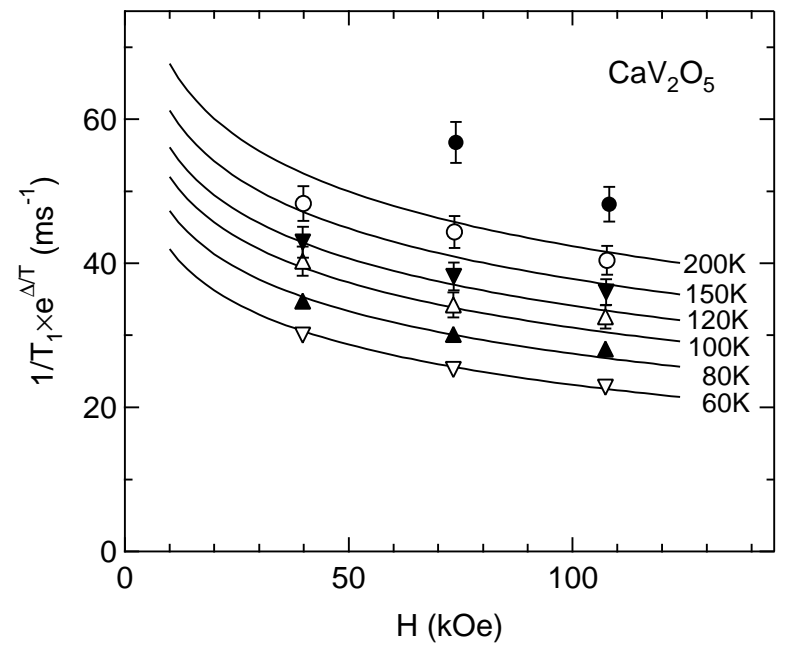

Fig. 4. Magnetic field dependence of $1 / T_{1}$. Solid line shows a fit to the free boson theory eq. (3.11). We take $\Delta \sim 562 \mathrm{~K}$.

where

$$
\rho_{m}=\sqrt{\frac{T \Delta}{2 \pi c^{2}}} \mathrm{e}^{-\beta(\Delta-m h)},
$$

and $L_{t}=\sqrt{\pi} \beta \mathrm{e}^{\Delta} / 3 T$. The scaling function is

$$
\begin{gathered}
\Phi_{2}(\Omega)=\ln \left(\frac{4 \sqrt{\pi} \mathrm{e}^{-\gamma}}{\Omega}\right)+\frac{\pi\left[\left(\sqrt{4+\Omega^{2}}+2\right)^{\frac{1}{2}}-\sqrt{\Omega}\right]^{2}}{4 \sqrt{\Omega}\left(\sqrt{4+\Omega^{2}}+2\right)^{\frac{1}{2}}} \\
+\ln \frac{\left[1+\Omega^{2} / \Psi^{2}(\Omega)\right]^{\frac{1}{2}}[1+\Psi(\Omega)]}{2 \Omega}, \\
\Psi(\Omega)=\left(\Omega \sqrt{1+\frac{\Omega^{2}}{4}}-\frac{\Omega^{2}}{2}\right)^{\frac{1}{2}},
\end{gathered}
$$

where $\gamma$ is Euler's constant. For the anisotropic two-leg ladder $1 / T_{1}$ is given by

$$
\frac{1}{T_{1}}=\frac{A_{\perp}^{2}}{4 \hbar} \frac{S_{ \pm}\left(\omega_{\mathrm{N}}\right)+S_{\mp}\left(\omega_{\mathrm{N}}\right)}{2} .
$$

In Fig. 5, we compare the theoretical results for $\Delta=$ $J_{\perp}-J_{\|} \sim 562 \mathrm{~K}$ and $c=\sqrt{\Delta J_{\|}} \sim 229 \mathrm{~K}$. The temperature dependence for the parabolic dispersion with these exchange parameters is shown in Fig. 3. Below $150 \mathrm{~K}$, the difference is negligible at the magnetic fields where $1 / T_{1}$ was measured. At $200 \mathrm{~K}$, we find a strong enhancement of $1 / T_{1}$ due to the magnon-magnon collision below $\sim 80 \mathrm{kOe}$. From these calculations, we found that the independent magnon approximation is good for the analysis of our experimental data except at $200 \mathrm{~K}$. Also we conclude that the discrepancy between the energy gap deduced from $1 / T_{1}$ and the NMR shift reported in the previous measurement ${ }^{13)}$ cannot be explained by this semiclassical theory.

\subsection{Effects of inter-ladder coupling}

We next discuss the effects of the inter-ladder coupling $J^{\prime}$. We numerically calculate $1 / T_{1}$ with eq. (3.10) in- 


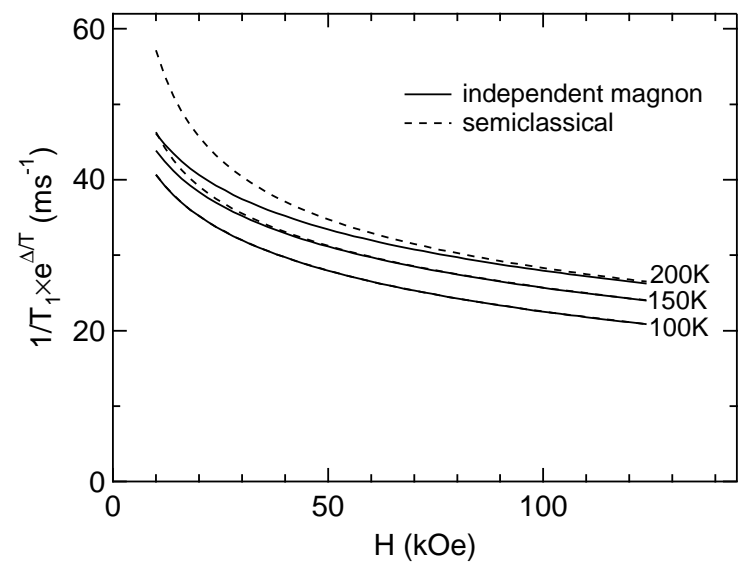

Fig. 5. Calculated magnetic field dependence of $1 / T_{1}$ by independent magnon approximation and semiclassical continuum theory with the parabolic dispersion with $\Delta=562 \mathrm{~K}$ and $c=229 \mathrm{~K}$.

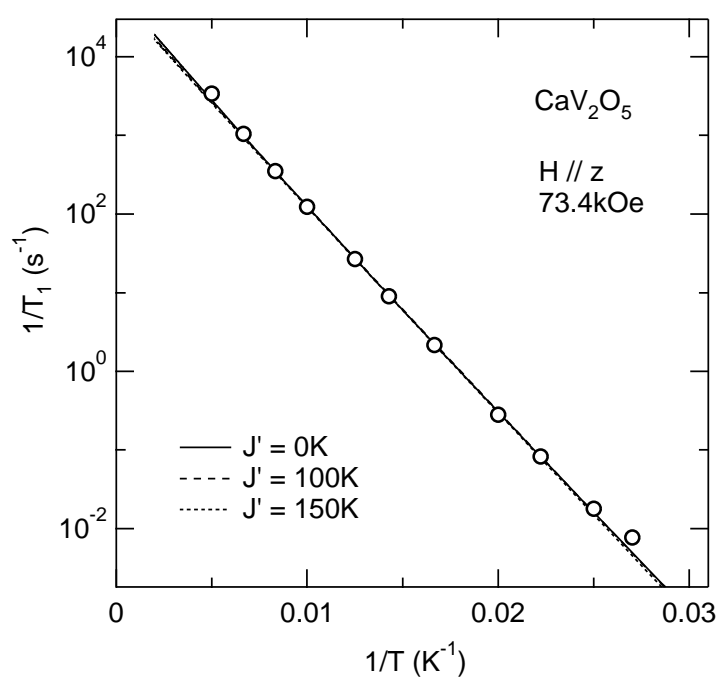

Fig. 6. Temperature dependence of $1 / T_{1}$ calculated with the inter-ladder coupling $J^{\prime}$. Solid line is for $J_{\perp}=655 \mathrm{~K}$, and $J_{\|}=$ $93 \mathrm{~K}$, and $\left|J^{\prime}\right|=0 \mathrm{~K}$, dashed line for $J_{\perp}=667 \mathrm{~K}, J_{\|}=88 \mathrm{~K}$, and $\left|J^{\prime}\right|=100 \mathrm{~K}$, and dotted line for $J_{\perp}=677 \mathrm{~K}, J_{\|}=81 \mathrm{~K}$, and $\left|J^{\prime}\right|=150 \mathrm{~K}$. Three lines are almost identical.

cluding $J^{\prime}$ and obtain good fits to the data with $0 \leq$ $\left|J^{\prime}\right| \lesssim 150 \mathrm{~K}$. The inter-ladder coupling affects $1 / T_{1}$ only weakly in this range of $J^{\prime}$; we can reproduce $1 / T_{1}$ with similar values of $J_{\perp}$ and $J_{\|}$even when $J^{\prime}$ changes. For example, $J_{\perp}=667 \mathrm{~K}$ and $J_{\|}=88 \mathrm{~K}$ for $\left|J^{\prime}\right|=100$ $\mathrm{K}$, and $J_{\perp}=677 \mathrm{~K}$ and $J_{\|}=81 \mathrm{~K}$ for $\left|J^{\prime}\right|=150 \mathrm{~K}$. $1 / T_{1}$ for these sets of exchange parameters is shown in Fig. 6; the difference is very small. Miyahara et al. calculated high temperature susceptibility by quantum Monte Carlo simulation and obtained an estimate $J_{\perp} \sim 670 \mathrm{~K}$, $0 \lesssim J_{\|} \lesssim 200 \mathrm{~K}$, and $J^{\prime}+J_{\|} \sim 110 \mathrm{~K}$. Their estimate agrees with our analysis of $1 / T_{1}$. For $\left|J^{\prime}\right| \gtrsim 150 \mathrm{~K}$, we found no range of $J_{\perp}$ and $J_{\|}$which reproduces the data.

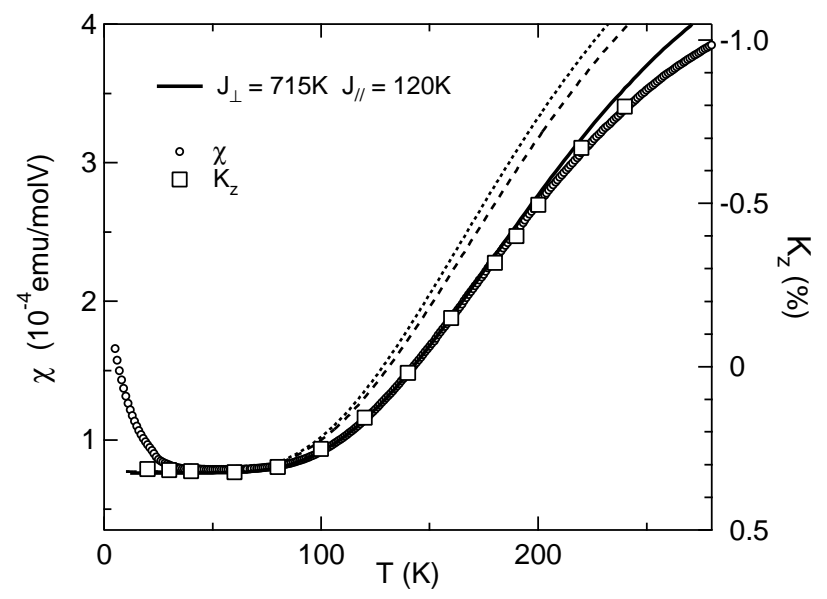

Fig. 7. Temperature dependence of magnetic susceptibility: solid line and dotted line are two-leg ladder models with $J_{\perp}=715 \mathrm{~K}$ and $J_{\|}=120 \mathrm{~K}$, and $J_{\perp}=655 \mathrm{~K}$ and $J_{\|}=93 \mathrm{~K}$, respectively. Dashed line is trellis lattice model with $J_{\perp}=667 \mathrm{~K}, J_{\|}=88 \mathrm{~K}$, and $J^{\prime}=100 \mathrm{~K}$. Small circle and large square are experimental results of magnetic susceptibility and NMR shift at the V sites, respectively. ${ }^{16)}$

\subsection{Magnetic susceptibility}

Onoda and Nishiguchi reported ${ }^{15)}$ that the measured susceptibility could be fitted by the dimer model with $J_{\perp}=664 \mathrm{~K}$

$$
\chi(T)=\frac{g^{2} \mu_{\mathrm{B}}^{2}}{T} \frac{\mathrm{e}^{-J_{\perp} / T}}{1+3 \mathrm{e}^{-J_{\perp} / T}} .
$$

However, our experimental result ${ }^{16)}$ cannot be fitted in the whole temperature range with $g=1.957$ measured by ESR. ${ }^{15)}$ This suggests that we need to consider interdimer couplings $J_{\|}$and $J^{\prime}$. In this section we compare the low-temperature susceptibility with the result of the strong-coupling expansion to check whether it can describe the temperature dependence of both the susceptibility and $1 / T_{1}$ consistently.

Troyer et al. gave an expression for the susceptibility of the two-leg ladder near the strong-coupling limit: ${ }^{24}$

$$
\begin{aligned}
\chi(T) & =\frac{g^{2} \mu_{\mathrm{B}}^{2}}{T} \frac{z(\beta)}{1+3 z(\beta)}, \\
z(\beta) & \equiv \frac{1}{2 \pi} \int_{-\pi}^{\pi} d k \mathrm{e}^{-\beta \epsilon_{k}} \\
& =\int_{0}^{\infty} d \epsilon \rho(\epsilon) \mathrm{e}^{-\beta \epsilon_{k}} .
\end{aligned}
$$

This expression is correct in both low and high temperature limits. For the cosine dispersion eq. (3.7), we obtain

$$
z(\beta)=\mathrm{e}^{-\beta J_{\perp}} I_{0}\left(\beta J_{\|}\right),
$$

where $I_{0}(x)$ is the modified Bessel function of the first kind and the susceptibility is given by eq. (4.7). Similarly we can calculate the susceptibility numerically for the trellis lattice including the inter-ladder coupling $J^{\prime}$ from eqs. (4.7) and (4.9) using the magnon density $\rho(\epsilon)$.

In Fig. 7 we show the experimental susceptibility. ${ }^{16)}$ To 
compare it to the theory, we have to obtain the spin susceptibility by subtracting the temperature-independent offset of the orbital and diamagnetic susceptibilities and magnetic impurity contributions (Curie tail). Since the experimental susceptibility is almost temperatureindependent between 30 and $70 \mathrm{~K}$, the susceptibility in this temperature range is a possible estimate of the sum of the orbital and diamagnetic susceptibilities. Then the increase below $30 \mathrm{~K}$ in decreasing temperature is attributed to impurity contributions. Since NMR shift is insensitive to such impurities, we can check if the observed temperature dependence of the susceptibility is intrinsic by comparing the susceptibility and the NMR shift. We plot the NMR shift at $\mathrm{V}$ sites ${ }^{16)}$ in Fig. 7. It is found that the NMR shift fits to the susceptibility well down to $40 \mathrm{~K}$ and that it is temperature-independent down to $20 \mathrm{~K}$. From this fitting we found that the observed temperature dependence above $\sim 50 \mathrm{~K}$ is intrinsic of the spin susceptibility and that the Curie tail is negligible above about $40 \mathrm{~K}$. We thereby obtain an estimate of the sum of the orbital and diamagnetic susceptibilities $\sim 7.7 \times 10^{-5} \mathrm{emu} / \mathrm{molV}$. Although the $K-\chi$ plot indicates that this 'residual susceptibility' is too large, ${ }^{16,27)}$ we use this estimate in the following analysis since we have no other reliable estimates.

We first calculate the susceptibility for the exchange parameters obtained from the analysis of $1 / T_{1}$; for the two-leg ladder model $J_{\perp}=655 \mathrm{~K}$ and $J_{\|}=93 \mathrm{~K}$, and for the trellis lattice model $J_{\perp}=667 \mathrm{~K}, J_{\|}=88 \mathrm{~K}$, and $J^{\prime}=100 \mathrm{~K}$. These parameters cannot reproduce the experimental susceptibility as shown in Fig. 7. Instead we can reproduce the low temperature susceptibility below $200 \mathrm{~K}$ with $J_{\perp}=715 \mathrm{~K}$ and $J_{\|}=120 \mathrm{~K}$ for the twoleg ladder model. The inter-ladder coupling $J^{\prime}$ affects the susceptibility only weakly and does not eliminate the disagreement between the exchange parameters from $1 / T_{1}$ and the susceptibility: for example we obtain almost the same temperature dependence with the parameters $J_{\perp}=$ $720 \mathrm{~K}, J_{\|}=120 \mathrm{~K}, J^{\prime}=100 \mathrm{~K}$. These results suggest the energy gap of $\Delta \sim 600 \mathrm{~K}$. The previous result of the NMR shift $\Delta \sim 460 \mathrm{~K}$ is an underestimate due to the large experimental error in the NMR shift measurement. ${ }^{13)}$

We thus found a discrepancy between the exchange parameters from $1 / T_{1}$ and the susceptibility. This may be reduced by i) a $1 / T_{1}$ calculation with higher-order of $J_{\|} / J_{\perp}$, ii) consideration of the off-diagonal part of the hyperfine interaction which was neglected, iii) a reliable estimate of the spin susceptibility, but we have no convincing explanations at present.

\section{$\S 5$. Conclusions}

We measured $1 / T_{1}$ at the $\mathrm{V}$ sites in the two-leg ladder antiferromagnet $\mathrm{CaV}_{2} \mathrm{O}_{5}$. We calculated $1 / T_{1}$ for the two-leg ladder with the strong-coupling expansion $\left(J_{\perp} \gg\right.$ $\left.J_{\|}\right)$within an independent magnon approximation, and compared the result with the experimental data. The comparison of the temperature dependence shows a good agreement and provides an estimate of both $J_{\perp} \sim 655(5)$ $\mathrm{K}$ and $J_{\|} \sim 93(5) \mathrm{K}$ as well as the energy gap $\Delta \sim 562(5)$ $\mathrm{K}$; the anisotropic exchange interactions $J_{\perp} \gg J_{\|}$was established. The magnetic field dependence of $1 / T_{1}$ is also reproduced within this approximation below $150 \mathrm{~K}$ $\sim \Delta / 4$. It is found that the magnon-magnon collision is negligible in this temperature range. We also studied the effect of inter-ladder coupling $J^{\prime}$. It is found that $J^{\prime}$ influences $1 / T_{1}$ only weakly and is in the range $0 \leq$ $\left|J^{\prime}\right| \lesssim 150 \mathrm{~K}$

We also calculated the magnetic susceptibility and compared with the previous experimental data. A discrepancy between the exchange parameters deduced from the magnetic susceptibility and $1 / T_{1}$ is found, although $1 / T_{1}$ can be satisfactorily understood within our calculation with the strong-coupling expansion.

\section{Acknowledgments}

We would like to thank Y. Itoh, J. Kikuchi, and H. Fukumoto for helpful discussions, and H. Tsunetsugu for useful comments.

1) For a review, E. Dagotto and T. M. Rice: Science 271 (1996) 618.

2) M. Azuma, Z. Hiroi, M. Takano, K. Ishida and Y. Kitaoka: Phys. Rev. Lett. 73 (1994) 3463.

3) M. Uehara, T. Nagata, J. Akimitsu, M. Takahashi, N. Môri, and K. Kinoshita: J. Phys. Soc. Jpn. 65 (1996) 2764.

4) K. Ishida, Y. Kitaoka, K. Asayama, M. Azuma, Z. Hiroi and M. Takano: J. Phys. Soc. Jpn. 63 (1994) 3222.

5) Y. Itoh and H. Yasuoka: J. Phys. Soc. Jpn. 66 (1997) 334.

6) J. Kishine and H. Fukuyama: J. Phys. Soc. Jpn. 66 (1997) 26.

7) S. Sachdev and K. Damle: Phys. Rev. Lett. 78 (1997) 943.

8) S. Sachdev: Quantum Phase Transitions (Cambridge University Press, Cambridge, 1999).

9) K. Damle and S. Sachdev: Phys. Rev. B 57 (1998) 8307.

10) M. Takigawa, T. Asano, Y. Ajiro, M. Mekata and Y. J. Uemura: Phys. Rev. Lett. 76 (1996) 2173.

11) Y. Furukawa, A. Iwai, K. Kumagai and A. Yakubovsky: J. Phys. Soc. Jpn. 65 (1996) 2393.

12) J. Kikuchi, T. Yamauchi and Y. Ueda: J. Phys. Soc. Jpn. 66 (1997) 1622.

13) H. Iwase, M. Isobe, Y. Ueda and H. Yasuoka: J. Phys. Soc. Jpn. 65 (1996) 2397

14) J. C. Bouloux and J. Galy: J. Solid State Chem. 16 (1976) 385.

15) M. Onoda and N. Nishiguchi: J. Solid State Chem. 127 (1996) 359.

16) T. Ohama, M. Isobe and Y. Ueda: J. Phys. Soc. Jpn. 69 (2000) 1574

17) S. Miyahara, M. Troyer, D.C. Johnston and K. Ueda: J. Phys. Soc. Jpn. 673918.

18) M. A. Korotin, I. S. Elfimov, V. I. Anisimov, M. Troyer, and D. I. Khomskii: Phys. Rev. Lett. 83 (1999) 1387.

19) M. A. Korotin, V. I. Anisimov, T. Saha-Dasgupta and I. Dasgupta: J. Phys. Condens. Matter. 12 (2000) 113.

20) A. Narath: Phys. Rev. 162 (1967) 320.

21) J. Sagi and I. Affleck: Phys. Rev. B 53 (1996) 9188.

22) T. Moriya: Prog. Theor. Phys. 16 (1956) 23.

23) T. Barnes, E. Dagotto, J. Riera and E. S. Swanson: Phys. Rev. B 44 (1993) 3196.

24) M. Troyer, H. Tsunetsugu and D. Würtz: Phys. Rev. B 50 (1994) 13515.

25) M. Reigrotzki, H. Tsunetsugu and T. M. Rice: J. Phys. Condens. Matter. 6 (1994) 9235.

26) G. M. Luke, Y. Fudamoto, M. J. P. Gingras, K. Kojima, M. Larkin, J. Merrin, B. Nachumi and Y. J. Uemura: J. Magn. Magn. Matter. 177-181 (1998) 754.

27) In ref. 28 Johnston et al. obtained large temperatureindependent susceptibility up to $750 \mathrm{~K}$ which consists of con- 
tributions of $\mathrm{CaV}_{3} \mathrm{O}_{7}$ as an impurity phase and another Curie component; the temperature dependence of these contributions are accidently cancelled out. This impurity contribution may partly explain the disagreement, but the estimated amount of the impurities seems too large. Similar large residual susceptibility was observed also in the charge ordered phase of $\alpha^{\prime}-\mathrm{NaV}_{2} \mathrm{O}_{5}$ in refs. 29 and 30 and we believe that it is intrinsic.

$28)$ D. C. Johnston, M. Troyer, S. Miyahara, D. Lidsky, K. Ueda, M. Azuma, Z. Hiroi, M. Takano, M. Isobe, Y. Ueda, M. A. Korotin, V. I. Anisimov, A. V. Mahajan and L. L. Miller: preprint (cond-mat/0001147).

29) T. Ohama, H. Yasuoka, M. Isobe and Y. Ueda: J. Phys. Soc. Jpn. 66 (1997) 3008.

30) T. Ohama, H. Yasuoka, M. Isobe and Y. Ueda: Phys. Rev. B 59 (1999) 3299. 